Original

\title{
Influence of periodontal treatment on objective measurement of masticatory performance
}

\author{
Luciano J. Pereira',2), Cátia M. Gazolla1), Isabela B. Magalhães1), \\ Matheus H. L. Dominguete1), Guilherme R. Vilela1), Paula M. Castelo3), \\ Leandro S. Marques ${ }^{1)}$ and Andries Van Der Bilt ${ }^{4}$ ) \\ 1)Department of Clinical Dentistry, University of Vale do Rio Verde, Três Corações, MG, Brazil \\ 2)Department of Physiology and Pharmacology, Federal University of Lavras, Lavras, MG, Brazil \\ 3)Department of Biological Sciences, Federal University of São Paulo, Diadema, SP, Brazil \\ 4)Department of Oral Physiology, Utrecht Medical Center, Utrecht, The Netherlands
}

(Received 18 October 2011 and accepted 7 March 2012)

\begin{abstract}
In a previous study, we evaluated the influence of periodontal treatment and physiological parameters on the subjective perception of mastication using the Oral Impact on Daily Performance Questionnaire (OIDP). In this study, we investigated the influence of periodontal treatment on the objective measures of masticatory performance in the same study sample. Patients with chronic periodontitis $(n=28)$ were examined on two occasions with a 45-day interval. Electrical activity of the masticatory muscles and maximum bite force were determined. In addition, masticatory performance was assessed using silicone impression blocks as test material. The median particle size of the chewed blocks was determined employing a sieving method. The number of teeth and the probing depth were also recorded. The probing depth was significantly reduced following treatment $(P<0.001)$. The median particle size of the chewed material was also reduced, which indicates a significant improvement in masticatory performance after treatment $(P<0.001)$. Bite force and muscle activity were significantly correlated both before and after treatment $(P<0.05)$. There was a negative correlation between masticatory performance and
\end{abstract}

Correspondence to Dr. Luciano José Pereira, Department of Physiology and Pharmacology, Federal University of Lavras UFLA, Lavras, MG, Mail Box 3037 CEP 37200-000, Brazil Tel: +55-35-3829-1471

E-mail: lucianojosepereira@dmv.ufla.br number of teeth $(P<\mathbf{0 . 0 5})$; moreover, individuals with a lower number of teeth exhibited poorer masticatory performance $(P=0.01)$. Periodontal treatment had a positive influence on masticatory performance 45 days after conservative treatment. (J Oral Sci 54, 151-157, 2012)

Keywords: electromyography; bite force; mastication; chronic periodontitis.

\section{Introduction}

Mastication is the first stage of the digestive process and results from rhythmic movements of the mandible by which food is shredded between the teeth (1). The mechanical shredding of food transforms it into smaller particles, thereby facilitating its enzymatic processing in the digestive system (2-4). The periodontal ligament provides information on the jaw relations and occlusal loads during food break down, adjusting the masticatory forces (5). Chronic periodontitis involves pathological detachment of collagen fibers from the cementum and junctional epithelium causing apical migration. Inflammatory events associated with connective tissue attachment loss also lead to the resorption of coronal portions of tooth supporting alveolar bone (6). These events can cause loss of receptors in the periodontal ligament, tooth mobility and reduced masticatory function (7). 
Masticatory function can be assessed by objective methods, such as evaluation of the electrical activity of the masticatory muscles (electromyography - EMG), masticatory performance and bite force (8-10). Masticatory performance is assessed by determining an individual's capability to fragment natural foods or test materials (11). Materials with uniform properties that allow reliable reproduction are ideal for evaluation of masticatory activity (8).

In a previous study conducted by our group in this sample, the number of teeth was found to have considerable influence on the perception of impact on mastication based on the Oral Impact on Daily Performance (OIDP) questionnaire (12). Furthermore, there was a significant negative correlation between the number of teeth and difficulty in eating before and after treatment. In this study performed on the same patients who participated in the previous study, we investigated the correlations among the objective measures of masticatory function (masticatory performance, bite force, masticatory muscle activity) and also the influence of periodontal treatment on the outcomes of masticatory performance.

\section{Materials and Methods}

The present study was approved by the Ethics Committee of the Vale do Rio Verde University, Três Corações, Minas Gerais, Brazil (process no. 0026.0.380.000-07). The participants signed terms of informed consent.

Characteristics of the subjects and the objective methods used for masticatory evaluation were described previously (12). Briefly, 283 individuals were initially screened for suitability based on the eligibility criteria during their first visit for dental treatment to the University Clinic. Thirty-two patients (18 women and 14 men) with chronic periodontitis were eligible for the study and were evaluated on two occasions with a 45-day interval. Twenty-eight patients completed both evaluations (age range 23 to 56 years; mean age: 37.9 years). All evaluated patients received dental treatment at the University Clinic, even those not taking part in this study, in the undergraduate practice.

The patients had at least 20 teeth and at least four occlusal units (one pair of molars in occlusion was considered two units and one pair of premolars in occlusion was considered one unit) (13). All patients were diagnosed to have chronic periodontitis based on the following criteria: attachment/bone loss (radiographic evaluation), bleeding upon probing, reddish discoloration of the gingiva and periodontal pockets with depths of $4 \mathrm{~mm}$ or more in at least four sites on different teeth.
Patients with diabetes or temporomandibular disorder, pregnant women, patients wearing dental appliances, those with teeth indicated for extraction, caries or pain (pulpitis) and those with implants were excluded from the study. Patients with removable dentures were instructed to remove them for the execution of the tests. Patients requiring surgical treatment were also excluded from the sample.

Periodontal status was assessed prior to and 45 days following treatment by the same examiner. The periodontal examination involved measuring pocket depth with a manual periodontal probe (Hu-Friedy PCP-UNC 15, Hu-Friedy, Chicago, IL, USA) to the nearest millimeter. The mean values of each patient were considered for statistical purposes (7).

All patients received basic periodontal treatment, which consisted of oral hygiene instructions, plaque control and scaling and root planing. The first treatment session started right after the first appointment for clinical examination and physiological measurements. The patients received quadrant-wise treatment of the whole dentition, under local anesthesia, at weekly intervals, in two to three sessions. Manual periodontal curettes were used (Hu-Friedy Gracey Standard Curettes SG 3/4, 11/12, 13/14, After Fives Curettes SAS 3/4, 11/12, 13/14, Hu-Friedy). The smoothness of the root surface was checked using a periodontal probe (Hu-Friedy PCP 11, Hu-Friedy) and an explorer (Hu-Friedy Wilkins-Tufts 17/23, Hu-Friedy) (14).

The probing depth was recorded before and after treatment by a trained examiner. This examiner was blinded to the study protocol and unaware of whether the patients were participating in the study in order to avoid bias on probing depth accuracy before and after treatment.

A signal conditioner (MCS-V2 EMG System do Brasil Ltd., São José dos Campos, Brazil) was used for electromyographic readings and determination of bite force. Muscle activity of both masseter and anterior portion of temporalis muscle were recorded using bipolar surface electrodes (Skintact Premier, ECG electrodes, $35 \times 50$ $\mathrm{mm}$, Leonhard Lang Ltd., Gloucestershire, UK) while participants performed maximal clenching. A stainless steel circular electrode was used as a reference electrode (ground electrode), placed on the back of the right wrist.

A force transducer of $12 \mathrm{~mm}$ that provides a signal corresponding to the applied compression force was used for measurement of the bite force, with an analog output reading of 0 to 5 volts and recording of the signal measured in Newton. The transducer was protected by a rubber cover, which made it soft to bite without any harm to the teeth. Besides, the cover had a mark for incisor 
placement in order to take all measurements at the same position. The transducer followed the dental arch format and was in contact with all teeth. Maximum voluntary bite force was measured bilaterally. Participants were instructed to bite as hard as possible on the bite force transducer. A verbal command was given to maintain clenching with maximal possible force for five seconds. The volunteer repeated the contraction twice, with a 5-min interval between readings. The means of root mean square (RMS) obtained on maximal clenching (during maximal bite force measurement) were used.

Masticatory performance was evaluated using the test material, Optosil (Optosil, Heraeuz Kulzer, Hanau, Germany) (15). The product was mixed and poured in acrylic molds with 5.6-mm cubic compartments under mechanical pressure. Each participant received 17 cubes (3.6 g), which were subjected to 20 mastication cycles. The subjects were then instructed to spit out the shredded particles onto filter paper and rinse the oral cavity with water to collect the remaining particles. The particles were rinsed with water, allowed to dry, removed from the filter paper, weighed and passed through a series of ten granulometric sieves with mesh size ranging from 5.6 to $0.50 \mathrm{~mm}$ connected in decreasing mesh size order upon a metal base. The particles were passed through the first sieve of the series and the set of sieves was maintained under vibration for $20 \mathrm{~min}$. The particles retained in each sieve were removed and weighed on an analytical scale with a precision of $0.001 \mathrm{~g}$. The distribution of the particles by weight was described by a cumulative function of the particles (15). A non-linear regression procedure was used to obtain estimates of the parameter (median particle size) describing this function. The median particle size $\left(\mathrm{X}_{50}\right)$ is the aperture of a theoretical sieve through which $50 \%$ of the test food particles can pass. A smaller median particle size denotes a better masticatory performance.
The error in the recording of EMG, bite force and masticatory performance was calculated by evaluation of two repeated measurements $\left(\mathrm{x}_{1}, \mathrm{x}_{2}\right)$ with a 14-day interval. The data from 10 control subjects of the same age were analyzed using the Dahlberg formula $(\mathrm{EM}=$ $\left.\sqrt{ } \Sigma\left(\mathrm{x}_{1}-\mathrm{x}_{2}\right)^{2} / 2 \mathrm{n}\right)$ and paired Student's $t$-test. There was no significant difference between the evaluations $(P>0.05)$ and the error of reproducibility was less than $10 \%$.

Statistical analysis was performed using the Sigma Stat 3.1 statistical package (Systat Software Inc., Chicago, USA), with the level of significance set at $5 \%$. Normality was assessed using the Shapiro-Wilk $W$-test. The Wilcoxon test was used to assess differences in the muscle electrical activity (EMG), bite force and masticatory performance values between sessions. Spearman's correlation coefficients were calculated for the determination of correlations between the variables. Multivariate logistic regression (backward stepwise procedure) was employed to test the association between the treatment (dependent variable; $0=$ prior to treatment; $1=$ following treatment) and the mastication parameters studied. The following were the independent variables: probing depth, median particle size, number of teeth, number of masticatory units and bite strength. These variables were introduced in the model and regressively removed (backward stepwise procedure); only those with a $P$-value $<$ 0.05 remained in the model.

The relative contributions of probing depth, number of teeth, number of masticatory units, muscle electrical activity and bite force to the masticatory performance variation between the two stages of periodontal treatment (before/after) were tested by multiple regression analysis with backward elimination. The effects of periodontal treatment stage (before/after) were captured by creating a dummy variable (0 or 1$)$.

Table 1 Mean values ( \pm standard deviation) for probing depth, masticatory performance, number of teeth, number of occlusal units, electromyographic activity (EMG) and maximum bite force $(\mathrm{N})$ in patients with chronic periodontitis before and 45 days after scaling and root planing (Wilcoxon test)

\begin{tabular}{lcccc}
\hline Physiological and clinical parameters & Before & After & Mean difference & P value \\
\hline Probing depth $(\mathrm{mm})$ & $4.4(1.1)$ & $3.7(0.9)$ & $0.7(0.7)$ & $<0.001^{*}$ \\
Masticatory performance $\left(\mathrm{X}_{50}-\mathrm{mm}\right)$ & $7.4(1.9)$ & $5.9(1.2)$ & $1.5(1.5)$ & $<0.001^{*}$ \\
Number of teeth & $28.3(2.4)$ & $28.3(2.4)$ & $0.0(0.0)$ & 1.000 \\
Number of occlusal units & $11.0(3.0)$ & $11.0(3.0)$ & $0.0(0.0)$ & 1.000 \\
EMG $(\mu \mathrm{V})$ (electromyographic activity) & $72.4(34.4)$ & $66.1(27.3)$ & $6.3(22.4)$ & 0.801 \\
Maximum bite force $(\mathrm{N})$ & $208.7(100.7)$ & $209.9(93.5)$ & $8.8(32.2)$ & 0.296 \\
\hline
\end{tabular}

$* P<0.05$ 
Table 2 Correlation between variables at two time-points: before and 45 days after periodontal treatment (Spearman's correlation coefficient)

\begin{tabular}{|c|c|c|c|c|c|c|c|}
\hline \multicolumn{3}{|c|}{ Physiological parameters } & \multirow{2}{*}{$\begin{array}{c}\mathrm{BF} \\
0.569\end{array}$} & \multirow{2}{*}{$\begin{array}{l}\text { EMG } \\
0.444\end{array}$} & \multirow{2}{*}{$\begin{array}{c}\text { PD } \\
0.489\end{array}$} & \multirow{2}{*}{$\begin{array}{c}\text { NT } \\
0.026^{*}\end{array}$} & \multirow{2}{*}{$\begin{array}{c}\mathrm{OU} \\
0.328\end{array}$} \\
\hline Masticatory & Before & $P$ & & & & & \\
\hline Performance & & $\mathrm{r}$ & -0.115 & -0.154 & -0.139 & -0.423 & -0.196 \\
\hline & After & $P$ & 0.228 & -0.267 & 0.172 & $0.049 *$ & 0.928 \\
\hline & & $\mathrm{r}$ & -0.240 & 0.178 & -0.271 & -0.409 & -0.018 \\
\hline \multirow[t]{4}{*}{ Bite Force } & Before & $P$ & - & $0.003 *$ & 0.649 & 0.462 & 0.194 \\
\hline & & $\mathrm{r}$ & - & 0.544 & 0.091 & 0.148 & 0.333 \\
\hline & After & $P$ & - & $0.001 *$ & 0.853 & 0.890 & 0.750 \\
\hline & & $\mathrm{r}$ & - & 0.574 & -0.037 & -0.027 & -0.064 \\
\hline \multirow[t]{4}{*}{ EMG } & Before & $P$ & - & - & 0.354 & 0.122 & 0.343 \\
\hline & & $\mathrm{r}$ & - & - & -0.186 & 0.305 & 0.190 \\
\hline & After & $P$ & - & - & 0.156 & 0.865 & 0.496 \\
\hline & & $\mathrm{r}$ & - & - & -0.281 & -0.034 & -0.137 \\
\hline
\end{tabular}

$* P<0.05 ; \mathrm{BF}-$ bite force; EMG - electromyographic activity; $\mathrm{PD}$ - probing depth; NT - number of teeth; $\mathrm{OU}$ - number of occlusal units

\section{Results}

Probing depth was significantly reduced following treatment $(P<0.001)$. The median particle size of Optosil particles $\left(\mathrm{X}_{50}\right)$ was also significantly smaller, indicating an improvement in masticatory performance $(\mathrm{P}<0.001)$ (Table 1).

The outcomes of all measured variables were correlated to one another before and after periodontal treatment (Table 2). There was a significant positive correlation between bite force and EMG both prior to and following treatment $(P<0.05)$. There was a negative correlation between masticatory performance and number of teeth $(P<0.05)$, which indicated smaller median particle sizes (better masticatory performance) for larger number of teeth.

The logistic regression model revealed that probing depth and masticatory performance were significantly associated with treatment. Thus, periodontal treatment reduced the probing depth and improved masticatory performance $(P<0.05)$ (Table 3$)$.

By means of backward stepwise multiple regression it was observed that the stage of periodontal treatment (dummy variable) was the most important factor that explained the masticatory performance change after periodontal treatment (19\%) (Table 4).

\section{Discussion}

In the present research, patients were subjected to non-surgical periodontal treatment. The re-evaluation of masticatory physiology was stipulated after a period of 45 days, which was determined based on the observation of significant improvements in probing depth following this period of time $(P<0.001)$. Moreover, multiple logistic regression revealed a significant association between probing depth and periodontal treatment. This finding demonstrates that conservative periodontal treatment with manual instruments (scaling and root planing) was capable of promoting significant improvements in clinical periodontal parameters (13) within a short period (45 days).

The patients achieved a significant improvement in masticatory performance following treatment, as was demonstrated by the better fragmentation of the Optosil blocks $(P<0.001)$. The improvement in masticatory performance $(7.4->5.9 \mathrm{~mm})$ is clinically relevant since patients could swallow finer food particles after treatment. The masticatory performance achieved by the patients after treatment was quite similar to that found in control subjects $(5.3 \mathrm{~mm})$ in a previous study at our laboratory (16). Individuals with a masticatory disorder tend to compensate by changing their diet to food with a softer consistency or by swallowing larger pieces (15). The former causes dietary imbalance due to inadequate consumption of essential nutrients and the latter may result in a reduction in the bio-availability of nutrients. In both cases, there is an increased risk of gastrointestinal disorders (17).

There was no association between masticatory performance and maximal bite force $(P>0.05$ - Table 4). Periodontal treatment is believed to have positively influenced performance, which is extremely dependent on the contact of tooth surfaces. An interesting association has previously been demonstrated between probing depth and premature contact, which reduces surface 
Table 3 Stepwise logistic regression used to test the association between masticatory parameters and the outcome (treatment)

\begin{tabular}{llccccccc}
\hline $\begin{array}{l}\text { Dependent } \\
\text { variable }\end{array}$ & $\begin{array}{c}\text { Independent } \\
\text { variable }\end{array}$ & Coef. & $P$ value & Odds ratio & CI & $\mathrm{R}^{2}$ & $\begin{array}{c}\mathrm{F} \\
P \text { value }\end{array}$ & Power of test \\
\hline \multirow{2}{*}{ Treatment } & constant & 2.463 & & & & & & \\
& PD & -0.197 & $<0.001^{*}$ & 0.250 & $0.10-0.66$ & & \\
& MP & -0.137 & $<0.001^{*}$ & 0.394 & $0.22-0.72$ & 0.37 & $<0.001$ & 0.99 \\
& EMG & -0.004 & 0.051 & 0.973 & $0.95-1.00$ & & & \\
\hline
\end{tabular}

* $P<0.05$; PD - probing depth; MP - masticatory performance; EMG - electromyographic activity; CI - confidence interval

Table 4 Stepwise linear regression used to test the relationship between masticatory performance and the studied independent variables

\begin{tabular}{|c|c|c|c|c|c|c|}
\hline \multirow{2}{*}{$\begin{array}{l}\text { Dependent } \\
\text { variable }\end{array}$} & \multirow{2}{*}{ Independent variables } & \multirow{2}{*}{ Coef. } & \multirow{2}{*}{$P$ value } & \multicolumn{3}{|c|}{ Significance of model } \\
\hline & & & & $\mathrm{R}^{2}$ & $P$ value & Power of the test \\
\hline \multirow{7}{*}{$\begin{array}{l}\text { Masticatory } \\
\text { performance } \\
(\times 50)\end{array}$} & constant & 7.385 & - & \multirow{7}{*}{0.19} & \multirow{7}{*}{$<0.001$} & \multirow{7}{*}{0.92} \\
\hline & Probing depth & - & 0.189 & & & \\
\hline & Number of teeth & - & 0.213 & & & \\
\hline & Number of masticatory units & - & 0.381 & & & \\
\hline & Muscle electrical activity (EMG) & - & 0.171 & & & \\
\hline & Bite force & - & 0.257 & & & \\
\hline & $d$ & -1.485 & $<0.001$ & & & \\
\hline
\end{tabular}

$d$ - dummy variable (stage of periodontal treatment)

contact between teeth and consequently, masticatory performance (18). To date, the influence of periodontal treatment on masticatory performance has not been longitudinally assessed.

Sensory signals from the periodontal ligament are important for the regulation of bite strength. In previous studies, alterations in probing depth and insertion level were found to be significantly correlated with a reduction in bite force $(7,19)$. An explanation for this is based on the belief that the loss of periodontal support results in compromised modulation of masticatory forces (5). However, although the bite force slightly increased after 45 days of periodontal treatment in the present study, the increase did not reach statistical significance $(P=0.296)$. The discrepancy in results between the studies cited and the present investigation may be related to methodological differences. In the present study, the assessment of bite force was carried out after 45 days of treatment, whereas the studies cited performed cross-sectional comparisons between individuals with different degrees of periodontal disease and a control group. Moreover, in the study by Alkan et al. (7), the patients with chronic periodontitis received periodontal treatment prior to the evaluation of bite force, whereas the initial assessment of bite force in the present study was performed prior to treatment. The results of the present investigation corroborate a previous population-based study in which periodontal status was not correlated to a reduction in bite force and was only influenced by the number of teeth present, gender and age (20).

It should be stressed that, although masticatory performance improved between the two evaluations and it is reported to be influenced by bite force in approximately $40 \%$ (21), the 45-day period is believed to have been insufficient for promoting an increase in muscle mass and, consequently, the bite force of individuals with periodontal disease. As discussed above, the improvement in the occlusal contact brought about by periodontal treatment may have had a positive effect on this variable. Another important issue is that bite force also depends on the number of teeth in occlusion (21). Patients with periodontal disease tend to experience tooth loss, which hinders comparisons to studies carried out on healthy patients. The fact that periodontal disease causes tooth mobility must also be taken into consideration. Thus, even after treatment, patients may be wary of applying maximal force on the transducer as a reflex developed to preserve the teeth.

The electromyographic activity of the masticatory muscles (EMG) was not influenced by periodontal treat- 
ment $(P>0.05)$. This finding corroborates a previous study, in which teeth with more than a 50\% reduction in periodontal support were equally able to support masticatory loads (22). A significant correlation was found between bite force and electromyographic activity before and after treatment $(P<0.05)$ as well as between masticatory performance and number of teeth $(P<0.05)$. These findings corroborate those described previously $(10,19)$.

In the present research, we observed a treatment response effect between extent/severity of periodontitis and masticatory function (Table 4). However, longitudinal randomized intervention trials with larger samples in patients against healthy controls are needed to improve the validity of these findings. However, comparisons of bite force, masticatory performance and EMG between the patient and control groups are susceptible to possible differences in number of teeth (21), facial pattern and muscle mass $(23,24)$. Comparisons among different subject groups may thus be made in homogenous subject groups, otherwise the results will be inconclusive.

Based on the results obtained in the studied sample, periodontal treatment had a positive influence on masticatory performance 45 days after conservative treatment.

\section{Acknowledgments}

The authors are grateful to the Research Support Foundation of the State of Minas Gerais (FAPEMIG), and Luciano Jose Pereira acknowledges the scholarship from the National Council for Scientific and Technological Development $(\mathrm{CNPq})$ during the execution of both parts of this study.

\section{References}

1. Pereira LJ, Duarte Gaviao MB, Van Der Bilt A (2006) Influence of oral characteristics and food products on masticatory function. Acta Odontol Scand 64, 193-201.

2. Hiiemae K, Heath MR, Heath G, Kazazoglu E, Murray J, Sapper D, Hamblett K (1996) Natural bites, food consistency and feeding behaviour in man. Arch Oral Biol 41, 175-189.

3. Julien KC, Buschang PH, Throckmorton GS, Dechow PC (1996) Normal masticatory performance in young adults and children. Arch Oral Biol 41, 69-75.

4. van der Bilt A, Engelen L, Pereira LJ, van der Glas HW, Abbink JH (2006) Oral physiology and mastication. Physiol Behav 89, 22-27.

5. Johansson AS, Svensson KG, Trulsson M (2006) Impaired masticatory behavior in subjects with reduced periodontal tissue support. J Periodontol 77, 1491-1497.

6. Savage A, Eaton KA, Moles DR, Needleman I (2009) A systematic review of definitions of periodontitis and methods that have been used to identify this disease. J Clin Periodontol 36, 458-467.

7. Alkan A, Keskiner I, Arici S, Sato S (2006) The effect of periodontitis on biting abilities. J Periodontol 77, 1442-1445.

8. Gambareli FR, Serra MD, Pereira LJ, Gavião MB (2007) Influence of measurement technique, test food, teeth and muscle force interactions in masticatory performance. J Text Stud 38, 2-20.

9. van der Bilt A, Engelen L, Abbink J, Pereira LJ (2007) Effects of adding fluids to solid foods on muscle activity and number of chewing cycles. Eur J Oral Sci 115, 198-205.

10. Rosa LB, Semprini M, Siéssere S, Hallak JE, Pagnano VO, Regalo SC (2009) Correlation between bite force and electromyographic activity in dentate and partially edentulous individuals. Electromyogr Clin Neurophysiol 49, 291-297.

11. Speksnijder CM, Abbink JH, van der Glas HW, Janssen NG, van der Bilt A (2009) Mixing ability test compared with a comminution test in persons with normal and compromised masticatory performance. Eur J Oral Sci 117, 580-586.

12. Pereira LJ, Gazolla CM, Magalhães IB, Ramos -Jorge ML, Marques LS, Gameiro GH, Fonseca DC, Castelo PM (2011) Treatment of chronic periodontitis and its impact on mastication. J Periodontol 82, 243-250.

13. Käyser AF (1981) Shortened dental arches and oral function. J Oral Rehabil 8, 457-462.

14. Ioannou I, Dimitriadis N, Papadimitriou K, Sakellari D, Vouros I, Konstantinidis A (2009) Hand instrumentation versus ultrasonic debridement in the treatment of chronic periodontitis: a randomized clinical and microbiological trial. J Clin Periodontol 36, 132-141.

15. Slagter AP, Bosman F, Van der Bilt A (1993) Comminution of two artificial test foods by dentate and edentulous subjects. J Oral Rehabil 20, 159-176.

16. de Matos LF, Pereira SM, Kaminagakura E, Marques LS, Pereira CV, van der Bilt A, Pereira LJ (2010) Relationships of beta-blockers and anxiolytics intake and salivary secretion, masticatory performance and taste perception. Arch Oral Biol $55,164-169$. 
17. N'gom PI, Woda A (2002) Influence of impaired mastication on nutrition. J Prosthet Dent 87, 667-673.

18. Harrel SK, Nunn ME (2009) The association of occlusal contacts with the presence of increased periodontal probing depth. J Clin Periodontol 36, 1035-1042.

19. Takeuchi N, Yamamoto $\mathrm{T}$ (2008) Correlation between periodontal status and biting force in patients with chronic periodontitis during the maintenance phase of therapy. J Clin Periodontol 35, 215-220.

20. Morita M, Nishi K, Kimura T, Fukushima M, Watanabe T, Yamashita F, Zhou R, Yang J, Xu X (2003) Correlation between periodontal status and biting ability in Chinese adult population. J Oral Rehabil 30, 260-264.

21. Fontijn-Tekamp FA, van der Bilt A, Abbink JH,
Bosman F (2004) Swallowing threshold and masticatory performance in dentate adults. Physiol Behav 83, 431-436.

22. Fernandes CP, Psarras V, Freitas LB, Ahlgren J (1994) Jaw-closing muscles: electromyographic activity of human subjects with reduced periodontal support. J Oral Rehabil 21, 165-175.

23. Pereira LJ, Gavião MB, Bonjardim LR, Castelo PM, van der Bilt A (2007) Muscle thickness, bite force, and craniofacial dimensions in adolescents with signs and symptoms of temporomandibular dysfunction. Eur J Orthod 29, 72-78.

24. Castelo PM, Gavião MB, Pereira LJ, Bonjardim LR (2007) Masticatory muscle thickness, bite force, and occlusal contacts in young children with unilateral posterior crossbite. Eur J Orthod 29, 149-156. 